\title{
Domain transformation approach to deterministic optimization of examination timetables
}

\author{
Siti Khatijah Nor Abdul Rahim ${ }^{1,2}$, Andrzej Bargiela ${ }^{3,4}$, Rong $\mathbf{Q u}^{3}$ \\ 1. School of Computer Science, University of Nottingham (Malaysia Campus), Selangor, Malaysia. 2. Faculty of Computer \\ and Mathematical Sciences, Universiti Teknologi MARA (Perak), Seri Iskandar, Perak, Malaysia. 3. School of Computer \\ Science, University of Nottingham, Nottingham, UK. 4. Institute of I nformatics, Krakow University of Technology, Krakow, \\ Poland
}

Correspondence: Siti Khatijah Nor Abdul Rahim. Address: School of Computer Science, University of Nottingham (Malaysia Campus), Jalan Broga, 43500, Semenyih, Selangor, Malaysia. Telephone: 60-389-248-000. Email:

khyx8skn@nottingham.edu.my.

Received: August 30, 2012

Accepted: October 30, 2012

Online Published: December 26, 2012

DOI : 10.5430/air.v2n1p122

URL: http://dx.doi.org/10.5430/air.v2n1p122

\section{Abstract}

In this paper we introduce a new optimization method for the examinations scheduling problem. Rather than attempting direct optimization of assignments of exams to specific time-slots, we perform permutations of slots and reassignments of exams upon the feasible (but not optimal) schedules obtained by the standard graph colouring method with Largest Degree ordering. The proposed optimization methods have been evaluated on the University of Toronto, University of Nottingham and International Timetabling Competition (ITC2007) datasets. It is shown that the proposed method delivers competitive results compared to other constructive methods in the timetabling literature on both the Nottingham and Toronto datasets, and it maintains the same optimization pattern of the solution improvement on the ITC2007 dataset. A deterministic pattern obtained for all benchmark datasets makes the proposed method more understandable to the users.

\section{Key words}

Examination timetabling, Optimization, Slots permutations, Reassigning exams

\section{I ntroduction}

Timetabling problems exist in numerous areas including educational timetabling, nurse rostering, transportation timetabling, sports timetabling and so on. Of all the timetabling problems, it was reported that the educational timetabling is one of the most widely studied problems ${ }^{[19]}$. Examples of educational timetabling include school timetabling, university course timetabling and university examination timetabling. In this study, we focus on the examination timetabling problem.

Exam timetabling represents a challenging computational problem due to the strong inter-dependencies between exams caused by the many-to-many relationship between students and exams. In the exam timetabling problems, the general objective is to generate feasible schedules, which satisfy basic constraints. It is very difficult to define a standard examination timetabling problem due to different constraints present in different academic institutions. Consequently, the 
effectiveness of the timetabling methods and algorithms is measured by their performance on a representative set of benchmark problems.

Two types of constraints defined in the timetabling literatures are:

\section{Hard Constraints}

These are the constraints that must be fulfilled at all times. The primary hard constraint is that two exams with a common student cannot be scheduled in the same slot. Another hard constraint that needs to be conformed is the room capacity; i.e. there must be enough space in a room to accommodate all students taking a given exam. A timetable which satisfies all hard constraints is called a feasible timetable.

\section{Soft Constraints}

Soft Constraints are not absolutely crucial but satisfaction of these constraints is beneficial to students and/or the institution. An example of a soft constraint is a requirement to space out exams taken by individual students as widely as possible across the examination session so that they have adequate revision time between their exams. Normally one cannot satisfy all soft constraints thus there is a need for a performance function measuring the degree of fulfillment of these constraints.

Feasible timetable can have exam orderings which do not satisfy many of the soft constraints. Consequently, a separate optimization process needs to be deployed to obtain better quality schedules. In general, optimization can be seen as a process that maximizes the benefits while minimizing the investment in resources that facilitate these benefits ${ }^{[24]}$. Optimization can be applied to many areas and disciplines. In the area of the examination scheduling, optimization methods include Constraint Logic Programming ${ }^{[15]}$ and Ant Colony ${ }^{[14]}$ etc, in addition to metaheuristics in recent years $^{[19]}$.

\subsection{Overview of the proposed optimization method}

This study has adopted an approach to the design of the exam schedule optimization that focuses on promoting understandability of the optimization process. To this end, we have avoided random exploration of the solution space such as that widely proposed in the literature, and have adhered to the deterministic evaluation of the search direction during the optimization process. In this study we used benchmark datasets (University of Toronto, University of Nottingham and International Timetabling Competition (ITC2007)). A key step in the proposed exam scheduling method was the pre-processing of constraints prior to the generation of a feasible timetable. This is done through the abstraction of essential features of the exam scheduling problem from the original student-exam data.

This data abstraction process constitutes a significant methodological contribution of this study, as it enables subsequent optimization of the examination schedule without the need to refer to the voluminous student-exam data in the course of the optimization. The approach was inspired by the insights from our previous studies on industrial process optimization ${ }^{[1,4,17]}$ and has been formalized as a Granular Computing methodology ${ }^{[5-7,16]}$. We propose that the transformation of the problem domain is an effective methodological approach to dealing with complex examination timetabling problems. By performing an appropriate pre-processing of the original student-exam data into an exam conflict matrix and a spread matrix we have mapped the original problem expressed in the multi-dimensional space of exams and students into a space with a reduced dimensionality of exams and exam-slots. We will refer to this approach to solving the timetabling problem as the domain transformation approach.

The exam conflict matrix is a square matrix of dimension equal to the number of exams. Entries in this matrix at position $(i, j)$ represent the number of students causing conflict between exams $i$ and $j$. The spread matrix ${ }^{[20]}$ is a square matrix of dimension $\mathrm{S}$, where $\mathrm{S}$ is a number of slots. Entries in the spread matrix at position $(p, q)$ represent the number of students 
who take both an exam from slot $p$ and slot $q$. The matrix is symmetrical with diagonal elements being omitted because students can take only one exam in any given exam slot.

The exam conflict matrix is generated by incrementing the value at position (i,j) by 1 for each student taking exams $i$ and $j$ when the student-exam list is traversed. Using this information (exam conflict matrix), together with an initial grouping of exams information through the early pre-processing stage, the spread matrix is then generated. This is done by incrementing the value at position $(p, q)$ by 1 if exam $p$ and exam $q$ are not grouped together in the early allocation process (means they are clashing).

The pre-processing of the original student-exam data into the exam conflict matrix and the spread matrix pays dividends in terms of minimizing the subsequent cross checking and cross referencing in the original data in the optimization process, thus speeds up the scheduling task. The essence of pre-processing is summarised by the pseudocode in Figure 1, and is elaborated in greater detail ${ }^{[20]}$.

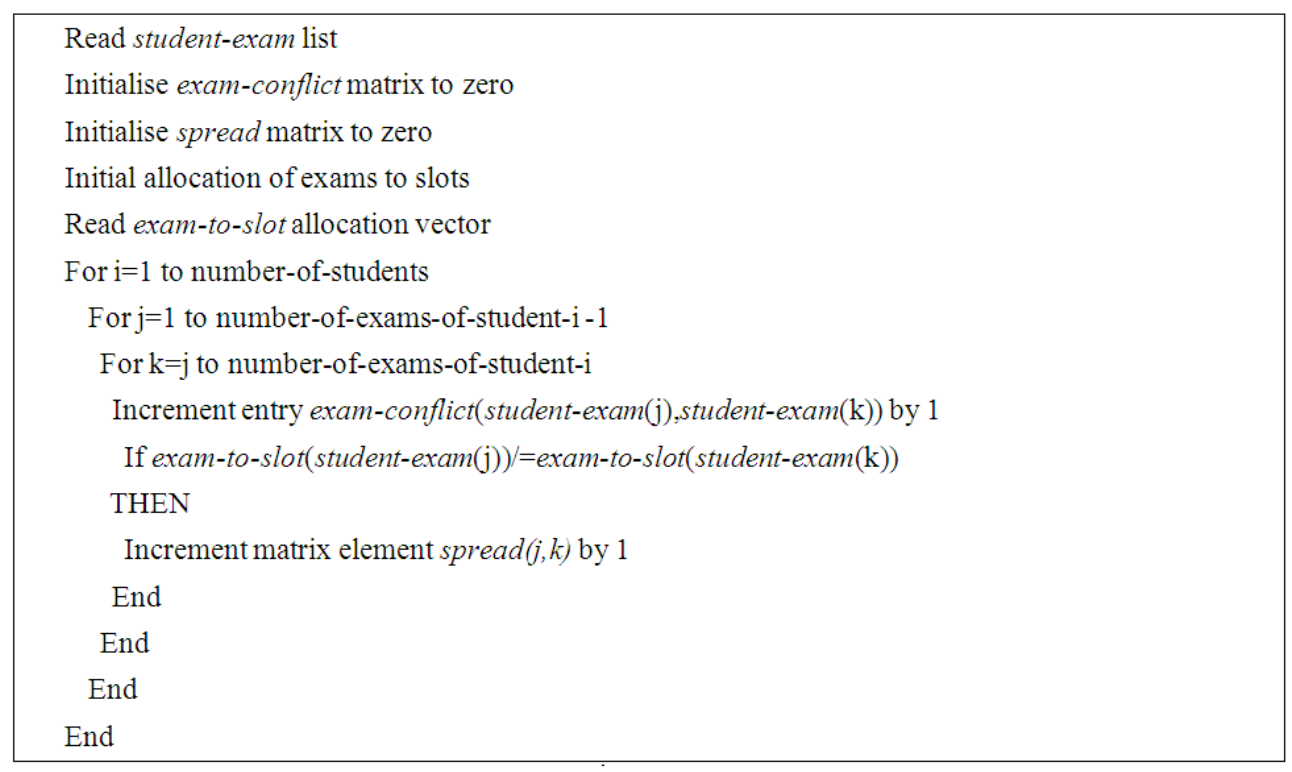

Figure 1. The pseudocode of the pre-processing

The proposed method proceeds in the following stages:

1) Problem domain transformation from student-exam to exam conflict and spread matrix data space (Figure 1)

2) Generation of a feasible solution (Figure 2)

3) Minimization of the overall slots conflicts (Figure 3)

4) Minimization of the schedule cost by slot swapping (Figure 5)

5) Minimization of the schedule cost by exam reallocation (Figure 7)

6) Repetition of stages 4 and 5 until there is no improvement in the schedule cost. 


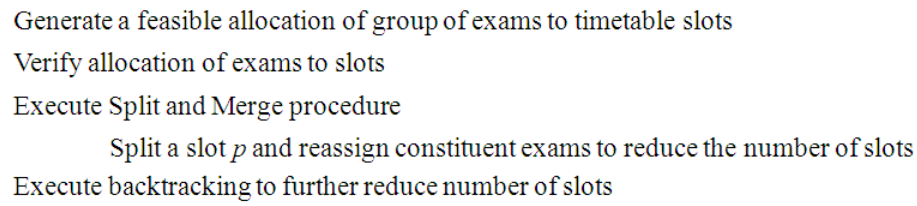

Figure 2. The pseudocode of the allocations of exams to slots

During the problem domain transformation (as in stage 1 above), initial allocation of exams to slots was done, i.e. grouping exams that are not conflicting in a group. This is based on the principle of an early allocation of exams with the highest number of conflicts to the available time slots. During this process, the number of conflicts of exams, which have not been scheduled yet, is recalculated to reflect the latest updated status of exams. Any unused slots are removed and provide a buffer-space for subsequent optimization. The output is an allocation flag, exam-to-slot vector which contains the slot number for all exams. At this point, the number of slots could be determined by the maximum value in the allocation flag.

Generation of a feasible solution is done by allocating a group of exams to timetable slots which are verified by calling a verification procedure. The process continues by calling the splitandmerge procedure to reallocate exams. By splitting a slot $p$ and reassigning constituent exams, the total number of slots may be reduced if every exam in slot $p$ can be allocated to some other slot, i.e. not in conflict with exams in other slots. We then perform backtracking to further reduce the number of slots if any reduction is possible. The process is illustrated by the pseudocode in Figure 2.

In this work, we develop optimization methods (see Section 2) to improve the initial feasible scheduled generated by the allocation method in Figure 2.

For each exam $i$ in the problem
Obtain the slot number (where it is allocated) from the allocation flag
Find the sum of the total slots conflicts, and set it as the lowest total slots conflicts
For each slot (except the slot for exam $i$ )
Calculate the new total slots conflicts by reassigning exam $i$ into a new slot
If the new total slots conflicts is lower than the lowest total slots conflicts
End Set the new total slots conflicts as the lowest total slots conflicts
Reassign exam $i$ to the slot that produced the lowest total slots conflicts
End

Figure 3. The pseudocode for minimizing total slots conflicts

\begin{tabular}{|c|c|c|c|c|c|c|c|c|c|c|}
\hline 0 & 1044 & 1108 & 918 & 948 & 708 & 628 & 47 & 222 & 7 & 1 \\
\hline 1044 & 0 & 1349 & 1119 & 1302 & 593 & 753 & 118 & 322 & 9 & 2 \\
\hline 1108 & 1349 & 0 & 1282 & 1198 & 575 & 786 & 166 & 342 & 9 & 3 \\
\hline 918 & 1119 & 1282 & 0 & 921 & 518 & 656 & 95 & 181 & 33 & 4 \\
\hline 948 & 1302 & 1198 & 921 & 0 & 684 & 733 & 159 & 194 & 33 & 5 \\
\hline 708 & 593 & 575 & 518 & 684 & 0 & 546 & 92 & 23 & 45 & 6 \\
\hline 628 & 753 & 786 & 656 & 733 & 546 & 0 & 79 & 140 & 43 & 7 \\
\hline 47 & 118 & 166 & 95 & 159 & 92 & 79 & 0 & 35 & 12 & 8 \\
\hline 222 & 322 & 342 & 181 & 194 & 23 & 140 & 35 & 0 & 25 & 9 \\
\hline 7 & 9 & 9 & 33 & 33 & 45 & 43 & 12 & 25 & 0 & 10 \\
\hline 1 & 2 & 3 & 4 & 5 & 6 & 7 & 8 & 9 & 10 & 0 \\
\hline
\end{tabular}

Figure 4. An example of a spread matrix 


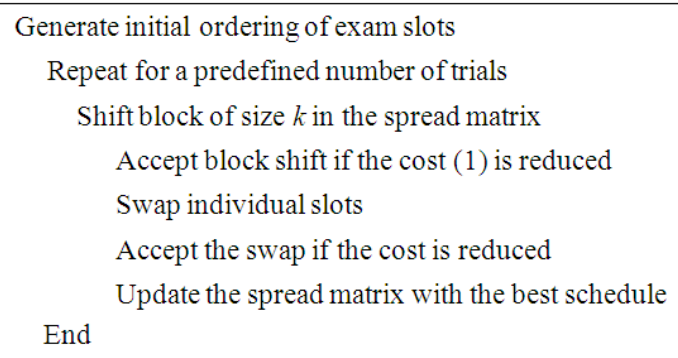

Figure 5. The pseudocode for the block and slot permutations

\begin{tabular}{|c|c|c|c|c|c|c|c|c|c|c|}
\hline 0 & 575 & 342 & 1198 & 9 & 1282 & 166 & 1108 & 786 & 1349 & 3 \\
\hline 575 & 0 & 23 & 684 & 45 & 518 & 92 & 708 & 546 & 593 & 6 \\
\hline 342 & 23 & 0 & 194 & 25 & 181 & 35 & 222 & 140 & 322 & 9 \\
\hline 1198 & 684 & 194 & 0 & 33 & 921 & 159 & 948 & 733 & 1302 & 5 \\
\hline 9 & 45 & 25 & 33 & 0 & 33 & 12 & 7 & 43 & 9 & 10 \\
\hline 1282 & 518 & 181 & 921 & 33 & 0 & 95 & 918 & 656 & 1119 & 4 \\
\hline 166 & 92 & 35 & 159 & 12 & 95 & 0 & 47 & 79 & 118 & 8 \\
\hline 1108 & 708 & 222 & 948 & 7 & 918 & 47 & 0 & 628 & 1044 & 1 \\
\hline 786 & 546 & 140 & 733 & 43 & 656 & 79 & 628 & 0 & 753 & 7 \\
\hline 1349 & 593 & 322 & 1302 & 9 & 1119 & 118 & 1044 & 753 & 0 & 2 \\
\hline 3 & 6 & 9 & 5 & 10 & 4 & 8 & 1 & 7 & 2 & 0 \\
\hline
\end{tabular}

Figure 6. Illustrations of spread matrix after permutations of slots are done

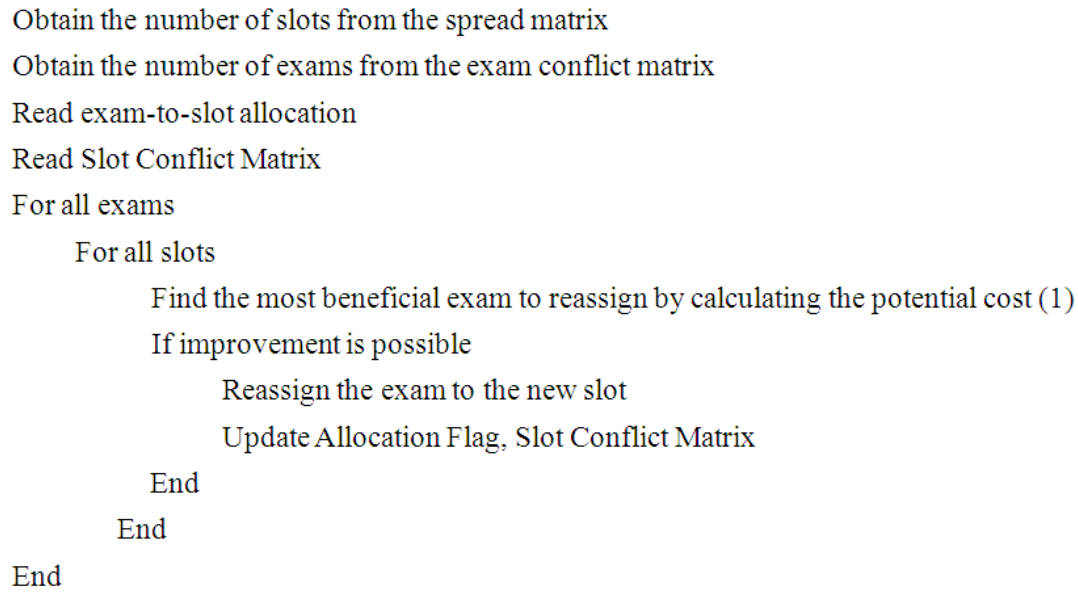

Figure 7. The pseudocode for the reassignments of exams

\section{Optimization methods}

The cost of the initial feasible schedule is normally fairly high. In order to minimize the cost, we perform the minimization of total slots conflicts, followed by further optimization on the initial schedule by the permutations of exam slots and the reassignment of exams between slots. 
The standard objective of exam scheduling is to minimize the cumulative inconvenience implied by the proximity of consecutive exams taken by individual students. This is measured by the cost function originally proposed in ${ }^{[12]}$ as follows:

$$
\frac{1}{T} \sum_{i=1}^{N-1} \sum_{j=i+1}^{N} s_{i j} w_{|\mathrm{pj}-\mathrm{pi}|}
$$

where $\mathrm{N}$ is the number of exams, $s_{i j}$ is the number of students enrolled in both exams $i$ and $j, p_{j}$ is the time slot where exam $j$ is scheduled, $p_{i}$ is the time slot where exam $i$ is scheduled and $\mathrm{T}$ is the total number of students. Based on this cost function, a student taking two exams that are $\left|p_{j}-p_{i}\right|$ slots apart, where $\left|p_{j}-p_{i}\right|=\{1,2,3,4,5\}$, leads to a cost of $16,8,4$, 2, and 1 , respectively.

The lower the cost obtained, the higher is the quality of the schedule, since the gap between two consecutive exams allows students to have extra revision time.

\subsection{Minimization of total slots conflicts}

The notion of a slot conflict is a generalization of the notion of exam conflict. We consider two exams $i$ and $j$ as being "in conflict” if there is a student that takes both exams. In a feasible schedule such exams are allocated to different exam slots. It is worth noting here that the conflict between exams is a binary property that does not increase in value if there are several students taking these two exams. Consequently, once we establish which exams are in conflict we do not need to be distracted, in the exam scheduling process, by the detailed student-exam data. This domain-transformation approach, motivated by the granular information processing paradigm ${ }^{[6]}$, provides a key advantage of the proposed exam scheduling.

Taking an even a broader view on the exam conflict, a novel contribution of this study is the consideration of the exam-slot conflict. Since every exam that is in conflict with the exam $i$ is allocated to some slot in the initial feasible solution, we can count the slots that contain conflicting exams. The exam-slot conflict is a binary property that does not increase in value if exam $i$ has several conflicting exams in one slot. Consequently, if all the exams conflicting with exam $i$ are allocated to the same slot, the exam-slot conflict for exam $i$ will be the lowest (equal to 1). On the other hand, if the conflicting exams are allocated to different slots, the exam-slot conflict for exam $i$ will be correspondingly higher (equal to the number of slots containing conflicting exams).

In order to guide our exam schedule optimization process, we use the total count of exam-slot conflicts as a measure of the ability to re-schedule exams between the slots. If the total count is high, it means that, on average, exams are in conflict with many slots and consequently there are few slots available for rescheduling. Conversely, if the total count is low, on average, there are more slots that can be used for re-scheduling of exams. To the best of our knowledge, the potential for rescheduling of exams has not been quantified in the literature so far despite it being a key factor enabling the improvement of the initial feasible schedule.

Recognizing the rationale for the maximization of the ability to reschedule individual exams between different slots, we start our optimization process by minimizing the total exam-slot conflict.

The procedure starts by taking the first exam $i$ in the dataset, and calculating the total slots conflicts. Next, we try to reassign exam $i$ to all other valid slots (i.e. not in conflict with exam $i$ ) and calculate the new total slots conflicts. A slot that could lead to a maximum reduction of total conflicts will be selected as a new slot for exam $i$. The procedure is repeated for all other exams in the problem. The pseudocode for minimizing slots conflicts is presented in Figure 3. 
By minimizing the total slots conflicts it is usually possible to reduce the cost of the exam schedule, as illustrated in Figure 9. However, we consider this stage primarily as the enhancement of the potential for the subsequent minimization of the cost of the schedule.

Nevertheless it is worth observing that although the cost formula (1) counts the spread of exams from the viewpoint of individual students, it is an integrative measure that is concerned with the average inter-exam spread. By reducing the total exam-slot conflict we achieve a greater packing of conflicting exams and, by implication, an increased possibility of separating the slots that have the largest number of conflicting exams.

\subsection{Minimization of cost by permutations of exams slots}

The second stage of the optimization is explicitly focused on the minimization of the cost function (1). The preparatory work of preparing the exam spread data structure, coupled with the maximization of the possibility of re-positioning (re-labeling) exam slots, brings dividends in terms of having a much smaller slot-optimization problem to consider while capturing the essence of the overall exam scheduling problem. Since the number of available exam slots is typically quite small, the optimization of the position of individual slots can be accomplished by the permutation of rows/columns of the spread matrix and the evaluation of the resulting cost (1).

Figure 4 presents an example of a spread matrix. The cost function (1) assigns a weight " 16 " to exams that are 1 slot apart (entries in the spread matrix $(1,2),(2,3),(3,4)$, etc.) and assigns a weight "8" to the exam of 2 slots apart (entries in the spread matrix $(1,3),(2,4),(3,5)$, etc.), and so on. To put it in a slightly more formal way, the weight " 16 " in the cost function is associated with the "first minor diagonal" entries of the spread matrix; weight "8" is associated with the "second minor diagonal" entries, etc. Assuming that the total number of students is 2749, the cost function (1) evaluates to:

$$
\begin{gathered}
{[[(1044+1349+1282+921+684+546+79+35+25) * 16]+} \\
{[(1108+1119+1198+518+733+92+140+12) * 8]+} \\
{[(918+1302+575+656+159+23+43) * 4]+} \\
{[(948+593+786+95+194+45) * 2]+} \\
[(708+753+166+181+33) * 1]] / 2749 \\
=56.99
\end{gathered}
$$

The potential for the reduction of this cost lies in the possibility of re-ordering the slots so as to replace the big numbers in first minor diagonal with the smaller entries that on subsequent minor diagonals.

The reordering of slots has been implemented as a simple greedy optimization process that involved swapping of the position of individual slots and also swapping of the position of groups of adjacent slots. If a swap operation improved the cost function (1) the swap was accepted and the exam slots were rearranged accordingly. Recognizing however that the greedy optimization may lead to local optima we have adopted a simple measure of restarting the optimization from several initial orderings of exam slots and picking the best solution from a pre-defined number of runs.

It is worth pointing out that while the optimization by permutation of slots does benefit from the prior minimization of the exam-slot conflict it does not affect the total count of the exam-slot conflict because the allocation of individual exams to slots does not change.

A single run of the optimization process outlined in Figure 5 on the spread matrix from Figure 4 results in the spread matrix presented in Figure 6. The large entries on the first minor diagonal in Figure 4 are replaced with much smaller values that were before positioned on higher order minor diagonals. The cost (1) after permutations of slots is: 


$$
\begin{gathered}
{[[(575+23+194+33+33+95+47+628+753) * 16]+} \\
{[(342+684+25+921+12+918+79+1044) * 8]+} \\
{[(1198+45+181+159+7+656+118) * 4]+} \\
{[(9+518+35+948+43+1119) * 2]+} \\
[(1282+92+222+733+9) * 1]] / 2749 \\
=31.81
\end{gathered}
$$

\subsection{Minimization of cost by reassignments of exams between slots}

In the third stage of the optimization, exams that make large contribution to the first minor diagonal entries of the reordered spread matrix are reassigned to slots represented by higher minor diagonals (preferably of order 6 or higher). Shifting an exam from one slot to another has a chain effect. Changes not only happen at the spread matrix level but also happen in the slot conflict matrix. Alteration of exam slots to reduce the cost function value could further reduce the overall conflict count or increase the value for the current solution. This is because the insertion of an exam to a slot can only happen if the slot contains exclusively exams that do not conflict with it. This action forces us to reevaluate the slot conflict count, which changes based on the slot location of all exams that is within the same chain as the shifted exam. The bigger the chain of the exam the bigger effect it will have to the conflict count. There are two methods of reassignment, single reassignments and group reassignments.

The single reassignments optimization move throughout the search space to identify an exam that has the biggest reduction to the cost function (1) if it were to be moved to other slots. The algorithm looks for a conflict-free slot which leads to the biggest cost reduction for all exams. The process of identifying the possible slots and calculating the cost function contribution is made simple by a data structure that combines the slot location and the penalty values generated by each exam for a slot.

The group reassignments optimization moves throughout the search space to identify an exam which could lead to the biggest reduction to the cost function. The optimization process evaluates the reduction from moving an exam to all other slots, and the best combination or total reduction configuration will be selected as the move that will be executed.

During the process of optimization the generated possible moves are evaluated against a history of moves two steps behind. The purpose of this is to eliminate possible cyclic moves in the optimization process. The group reassignments move exams to another slot if a reduction can be obtained. This may push other exam(s) out of the selected slot to alternative slots. There is a possibility that these exams switch slots and keeps on giving an improvement to the Carter cost thus keeping the optimization process ongoing. This will not stop if these two exams keep on exchanging slots. This kind of moves must be identified to eliminate infinite swapping. The process continues until there is no more improvements available and the number of iterations is more than half of the number of exams.

Both the single and group reassignments start by evaluating each exam one at a time and look for possible slot locations that could accept the exam without any clashes. The main difference is that the evaluation criteria to shift and the number of exams for every shift. The single reassignment will end up with solutions within the local optima due to the minor changes made to the initial placement of exams.

However, the group optimization has the possibility of moving solutions out from the local optima, resulting in a better result. This is due to the larger changes made at each step where all exams are evaluated and shifted at each cycle.

The effect of reassignments on the schedule is illustrated in Figure 9. It is shown that cost (1) has been reduced at the expense of some increase of the total slots conflicts. 
The pseudocode for the reassignment process is outlined in Figure 7.

\section{Experiments, results and discussions}

The study was performed on all 13 datasets in the Toronto benchmark repository [ftp://ftp.mie.utoronto.ca/pub/ carter/testprob] and also on the Nottingham dataset [http://www.cs.nott.ac.uk/ rxq/files/Nott.zip] that, for the sake of comparability with other studies, is considered here as an uncapacitated scheduling problem, where the total room capacity in each slot is not considered.

According to ${ }^{[19]}$, to indicate the density of the conflicting exams in each of the instances, a Conflict Matrix $\mathrm{C}$ is defined where each element $c_{i j}=1$ if exam $i$ conflicts with exam $j$ (have common students), or $c_{i j}=0$ otherwise. The Conflict Density represents the ratio between the number of elements of value " 1 " to the total number of elements in the conflict matrix.

The characteristics of all the datasets are listed in Table 1 . For the Toronto dataset, according to ${ }^{\text {[19] }}, 8$ out of 13 problem instances exist in 2 versions. We will use version I of the datasets which are widely tested by other researchers.

Table 1. Benchmark Datasets Used in the Timetabling Research

\begin{tabular}{|c|c|c|c|c|c|}
\hline Name of Dataset & No of Exams & No of Students & No of Enrollments & Required No of slots & Conflict Density \\
\hline $\begin{array}{l}\text { Nott (Nottingham a } \\
\text { or Nottingham b }\end{array}$ & 800 & 7896 & 33997 & 23 & 0.03 \\
\hline car-s-91 (I) & 682 & 16925 & 56877 & 35 & 0.13 \\
\hline car-s-91 (II) & 682 & 16925 & $56242 / 56877$ & 35 & 0.13 \\
\hline car-f-92 (I) & 543 & 18419 & 55522 & 32 & 0.14 \\
\hline car-f-92 (II) & 543 & 18419 & $55189 / 55522$ & 32 & 0.14 \\
\hline ear-f-83(I) & 190 & 1125 & 8109 & 24 & 0.27 \\
\hline ear-f-83(II) & 189 & 1108 & 8014 & 24 & 0.27 \\
\hline hec-s-92(I) & 81 & 2823 & 10632 & 18 & 0.42 \\
\hline hec-s-92(II) & 80 & 2823 & 10625 & 18 & 0.42 \\
\hline kfu-s-93 & 461 & 5349 & 25113 & 20 & 0.06 \\
\hline lse-f-91 & 381 & 2726 & 10918 & 18 & 0.06 \\
\hline pur-s-93 (I) & 2419 & 30029 & 120681 & 42 & 0.03 \\
\hline pur-s-93 (II) & 2419 & 30029 & $120686 / 120681$ & 42 & 0.03 \\
\hline rye-f-92 & 486 & 11483 & 45051 & 23 & 0.07 \\
\hline sta-f-83(I) & 139 & 611 & 5751 & 13 & 0.14 \\
\hline sta-f-83(II) & 138 & 549 & 5689 & 35 & 0.14 \\
\hline tre-s-92 & 261 & 4360 & 14901 & 23 & 0.18 \\
\hline uta-s-92(I) & 622 & 21266 & 58979 & 35 & 0.13 \\
\hline uta-s-92(II) & 638 & 21329 & 59144 & 35 & 0.13 \\
\hline ute-s-92 & 184 & 2749 & 11793 & 10 & 0.08 \\
\hline yor-f-83 (I) & 181 & 941 & 6034 & 21 & 0.29 \\
\hline yor-f-83 (II) & 180 & 919 & 6012 & 21 & 0.29 \\
\hline
\end{tabular}

For each dataset, as explained previously, the experiment starts by the pre-processing on the student-exam data, running the allocation method to generate a feasible solution and followed by the backtracking process to reduce the number of slots. Optimization then takes place. Recall that the optimization involves three processes: 1) Minimisation of the total slots conflicts, 2) Minimisation of cost by permutations of exams slots, and 2) Minimisation of cost by reassignments of exams. 
Accepting that the Permutations of Exams Slots based on the greedy optimization may lead to local optima, we have investigated the sensitivity of this optimization to the number of starting points so as to ensure sufficient exploration of the search space and promote the convergence to the global optimum. However, no claim is made about the exhaustive exploration of the search space and instead we offer the plots of the convergence trajectories in the "exam conflict schedule cost” space as an indication of the robust performance of the proposed method.

\subsection{Different parameters for permutations of slots}

We have tested different combinations of parameters in order to find the ideal or sufficient combinations that would lead to local optima. We have used 6, 9 and 12 starting points; and performed experiments for 4, 8, 10 and 12 iterations. All combinations were tested and the results for all datasets are recorded in Table 2.

Table 2. Optimized number of starting points and repetitions of the permutations of exam slots for different benchmark problems

\begin{tabular}{llllll}
\hline Dataset & $\begin{array}{l}\text { Carter Cost Before } \\
\text { Permutations } \\
\text { of Slots (Before } \\
\text { Optimizations) }\end{array}$ & $\begin{array}{l}\text { Number of Starting } \\
\text { Points Providing } \\
\text { Best (local) } \\
\text { Optimum }\end{array}$ & $\begin{array}{l}\text { Number of Repetitions } \\
\text { Providing Best (local) } \\
\text { Optimum }\end{array}$ & $\begin{array}{l}\text { Carter Cost After } \\
\text { Permutations of } \\
\text { Slots }\end{array}$ & $\begin{array}{l}\text { CPU Time } \\
\text { (seconds) }\end{array}$ \\
\hline Nott & 36.25 & 6 & 6 & 10.74 & 15.95 \\
car-s-91 & 10.73 & 9 & 6 & 6.36 & 201.50 \\
car-f-92 & 8.85 & 12 & 4 & 5.29 & 101.72 \\
ear-f-83 & 67.84 & 6 & 4 & 39.54 & 18.59 \\
hec-s-92 & 16.81 & 6 & 4 & 11.49 & 10.77 \\
kfu-s-93 & 34.52 & 12 & 4 & 15.91 & 18.25 \\
lse-f-91 & 23.32 & 6 & 4 & 14.11 & 9.14 \\
pur-s-93 & 13.85 & 9 & 6 & 6.64 & 27.34 \\
rye-f-92 & 25.97 & 6 & 4 & 12.34 & 18.70 \\
sta-f-83 & 195.53 & 9 & 6 & 9.75 & 6.05 \\
tre-s-92 & 13.66 & 6 & 4 & 4.28 & 14.42 \\
uta-s-92 & 6.75 & 9 & 4 & 30.85 & 149.08 \\
ute-s-92 & 38.35 & 6 & 4 & 39.94 & 34.45 \\
yor-f-83 & 51.07 & 6 & & & \\
\hline
\end{tabular}

The study indicated that a combination of 12 starting points and 6 iterations provided the best (sub-optimal) results on the benchmark dataset and that the increase of the number of iterations did not produce any improvement of cost. In order to enhance even further the exploration of the search space we have adopted 24 random starting points and 6 iterations in all subsequent experiments. Please note that this is possible because of the relatively computationally inexpensive optimization of the slot ordering.

\subsection{The final results and discussions}

The final results for all the datasets utilizing 24 number of starting points and 6 iterations are therefore presented in Table 3. Using the data gathered from the experiments on all the datasets, we have plotted graphs for the cost (1) versus the Total Slots Conflicts in Figure 8.

We can observe that the general pattern of the lines (graphs) consists of 3 stages, we name them as section (a), (b) and (c) from right to left, as illustrated in Figure 9. Decrease of the total slots conflicts in section (a) is typically (but not necessarily) coupled with the decrease of the exam schedule cost. In the second stage, in section (b), the exam schedule cost is reduced without any augmentation of the total slots conflicts. The third stage, represented in section (c) involves reassignment of exams that reduces the exam schedule cost but increase the total slots conflicts. Please note however that 
for some datasets (hec-s-92(I), lse-f-91, pur-s-93(I) and rye-f-92), only section (b) and (c) can be seen in the graph because they managed to record the best results even without running the minimization of the slots conflicts procedure.

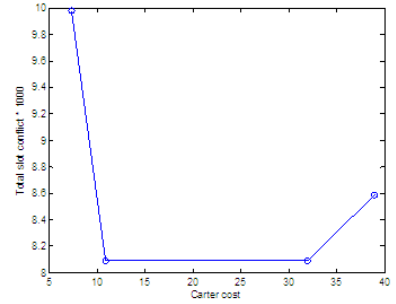

nott

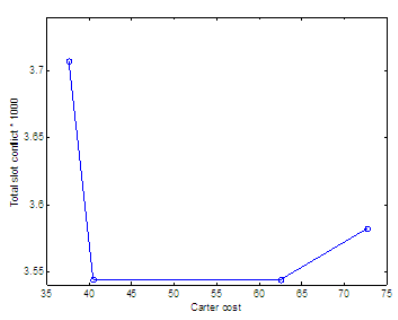

ear-f-83(I)

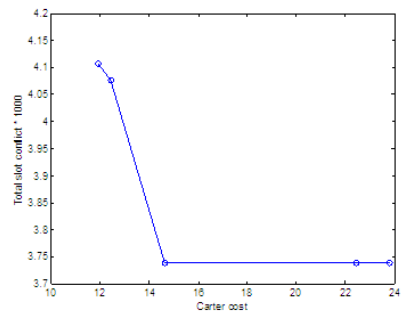

lse-f-91

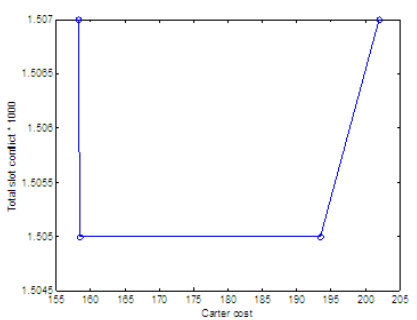

sta-f-83(I)

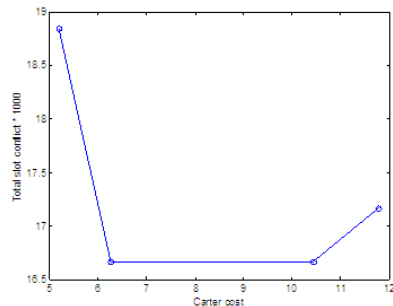

car-s-91 (I)

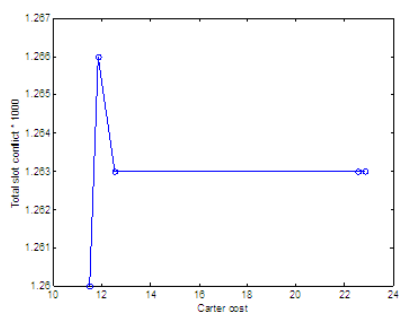

hec-s-92(I)

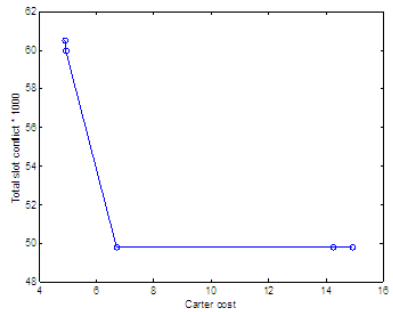

pur-s-93 (I)

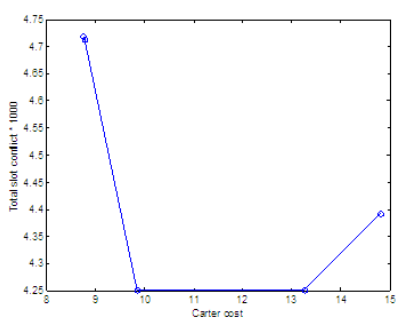

tre-s-92

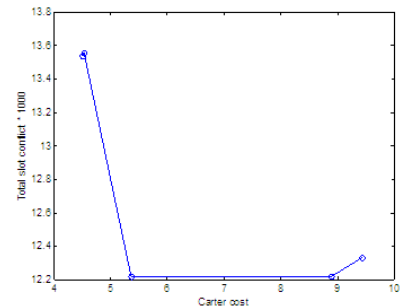

car-f-92 (I)

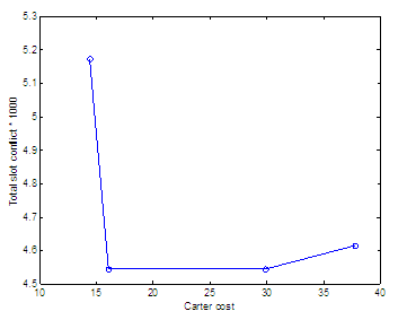

kfu-s-93

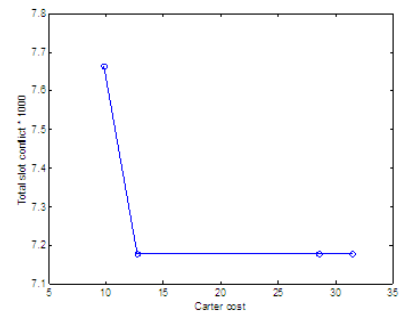

rye-f-92

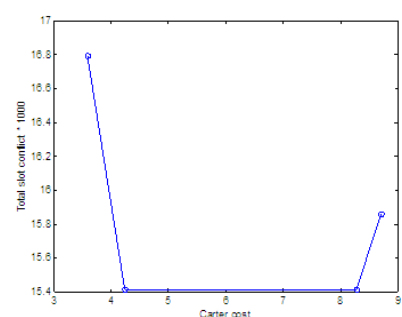

uta-s-92(I)
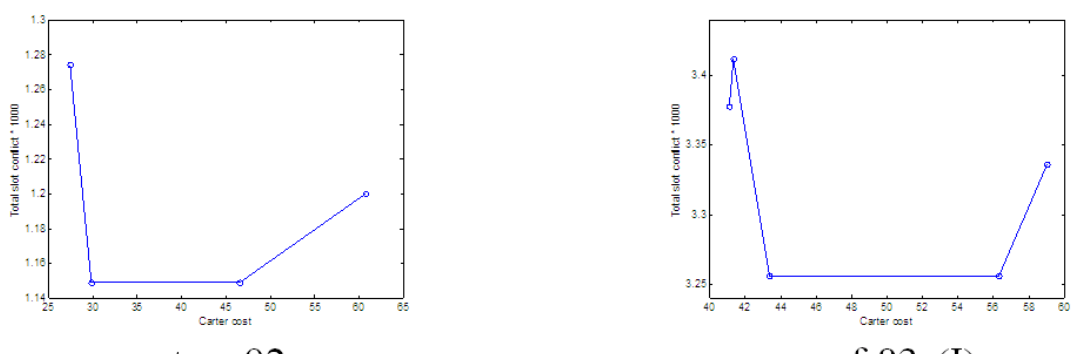

ute-s-92

yor-f-83 (I)

Figure 8. Cost (1) vs. the Total Slots Conflicts For Nott and Toronto Dataset 


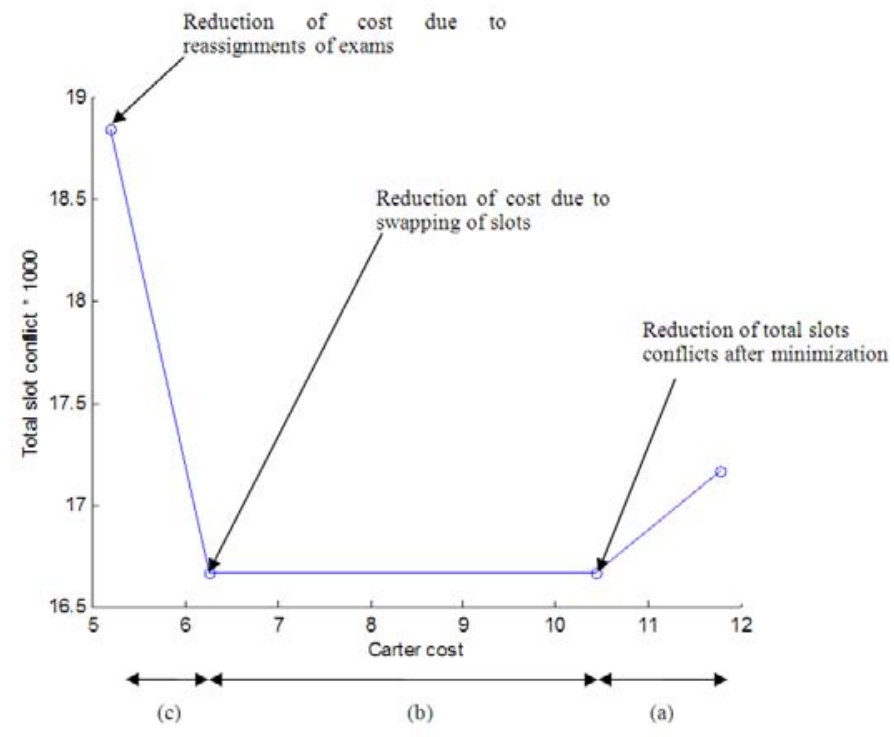

Figure 9. The Predicted Pattern of the Graph with the Proposed Approach

Table 3. Computational Results of the Proposed Approach Applied to the Toronto Dataset

\begin{tabular}{|c|c|c|c|c|c|c|c|c|c|c|c|c|c|}
\hline Dataset & $\begin{array}{l}\text { Re- } \\
\text { quired } \\
\text { No of } \\
\text { Slots }\end{array}$ & $\begin{array}{l}\text { Initial } \\
\text { Cost }\end{array}$ & $\begin{array}{l}\text { Total } \\
\text { Slots } \\
\text { Conflicts }\end{array}$ & $\begin{array}{l}\text { Minimiza- } \\
\text { tion of Slots } \\
\text { Conflicts }\end{array}$ & $\begin{array}{l}\text { Total } \\
\text { Slots } \\
\text { Conflicts }\end{array}$ & $\begin{array}{l}\text { Cost Before } \\
\text { Further } \\
\text { Optimiz- } \\
\text { ation }\end{array}$ & $\begin{array}{l}\text { Reassign- } \\
\text { ment } \\
\text { (G:group } \\
\text { /S:single) }\end{array}$ & $\begin{array}{l}\text { Cost } \\
\text { After } \\
\text { Swap } \\
1\end{array}$ & $\begin{array}{l}\text { Cost } \\
\text { After } \\
\text { Reassign } \\
\text { I }\end{array}$ & $\begin{array}{l}\text { Total } \\
\text { Slots } \\
\text { Conflicts }\end{array}$ & $\begin{array}{l}\text { Cost } \\
\text { After } \\
\text { Swap } \\
\text { II }\end{array}$ & $\begin{array}{l}\text { Cost } \\
\text { After } \\
\text { Reassign } \\
\text { II }\end{array}$ & $\begin{array}{l}\text { Total } \\
\text { Slots } \\
\text { Conflicts }\end{array}$ \\
\hline Nott & 23 & 38.99 & 8589 & YES & 8090 & 31.95 & S & 10.94 & 7.34 & 9979 & 7.34 & 7.34 & 9979 \\
\hline car-s-91 (I) & 35 & 11.77 & 17169 & YES & 16665 & 10.43 & S & 6.26 & 5.19 & 18847 & 5.19 & 5.19 & 18847 \\
\hline car-f-92 (I) & 32 & 9.43 & 12332 & YES & 12217 & 8.89 & G & 5.36 & 4.52 & 13558 & 4.52 & 4.49 & 13535 \\
\hline ear-f-83(I) & 24 & 72.69 & 3582 & YES & 3544 & 62.57 & S & 40.45 & 37.57 & 3707 & 37.57 & 37.57 & 3707 \\
\hline hec-s-92(I) & 18 & 22.83 & 1263 & NO & 1263 & 22.55 & G & 12.52 & 11.85 & 1266 & 11.62 & 11.47 & 1260 \\
\hline kfu-s-93 & 20 & 37.79 & 4616 & YES & 4544 & 29.89 & G & 16.06 & 14.36 & 5174 & 14.36 & 14.36 & 5174 \\
\hline lse-f-91 & 18 & 23.77 & 3739 & NO & 3739 & 22.42 & S & 14.63 & 12.41 & 4077 & 12.35 & 11.90 & 4107 \\
\hline pur-s-93 (I) & 42 & 14.91 & 49821 & NO & 49821 & 14.27 & G & 6.69 & 4.92 & 60005 & 4.92 & 4.88 & 60532 \\
\hline rye-f-92 & 23 & 31.50 & 7178 & NO & 7178 & 28.55 & G & 12.68 & 9.80 & 7664 & 9.80 & 9.80 & 7664 \\
\hline sta-f-83(I) & 13 & 201.95 & 1507 & YES & 1505 & 193.47 & G & 158.43 & 158.25 & 1507 & 158.25 & 158.25 & 1507 \\
\hline tre-s-92 & 23 & 14.81 & 4392 & YES & 4251 & 13.25 & G & 9.84 & 8.77 & 4714 & 8.77 & 8.74 & 4719 \\
\hline uta-s-92(I) & 35 & 8.71 & 15859 & YES & 15416 & 8.28 & S & 4.24 & 3.59 & 16792 & 3.59 & 3.59 & 16792 \\
\hline ute-s-92 & 10 & 60.71 & 1200 & YES & 1149 & 46.57 & G & 29.82 & 27.37 & 1274 & 27.37 & 27.37 & 1274 \\
\hline yor-f-83 (I) & 21 & 59.04 & 3336 & YES & 3256 & 56.31 & G & 43.36 & 41.35 & 3412 & 41.27 & 41.10 & 3378 \\
\hline
\end{tabular}

In order to obtain better schedules with lower cost, we have performed the permutations of exam slots and the reassignment of exams between slots.

In order to test the flexibility and ensure that our approach can work well on other datasets, we have further tested the methods on the International Timetabling Competition 2007 (ITC2007) dataset that can be obtained from [http://www.cs. qub.ac.uk/itc2007/Login/SecretPage.php]. The ITC2007 dataset contains additional constraints including room capacities, period utilization, period related and room related in the objective function. Table 4 presents some important characteristics of the ITC2007 benchmark dataset. The results obtained for all exams in this dataset can be seen in Table 5 . The cost (1) vs. the Total Slots Conflicts is plotted in Figure 10.

From all the results presented in Table 3, it is clear that the optimization of the initial feasible timetable resulted in an improved exam timetable with lower cost (1). For instance, for the "Nott" dataset, a reduction of the cost from 38.99 to 
10.94 has been obtained after the permutations of exams slots on the initial schedule. The cost was further improved to 7.34 after the reassignments of exams.

Table 4. The characteristics of the ITC2007 dataset

\begin{tabular}{lllll}
\hline Name of Dataset & No of Exams & No of Students & Required No of Slots & Conflict Density \\
\hline Exam1 & 607 & 7891 & 54 & 5.05 \\
Exam2 & 870 & 12743 & 40 & 1.17 \\
Exam3 & 934 & 16439 & 36 & 2.62 \\
Exam4 & 273 & 5045 & 21 & 15.0 \\
Exam5 & 1018 & 9253 & 42 & 0.87 \\
Exam6 & 7909 & 16 & 6.16 \\
Exam7 & 1096 & 14676 & 80 & 1.93 \\
Exam8 & 598 & 7718 & 80 & 4.55 \\
\hline
\end{tabular}

For the ITC2007 dataset, results reported in Table 5 also show significant reductions of the exam schedule cost compared to the cost of the original feasible schedule. For example, the cost of the exam schedule evaluated for the benchmark problem Exam8 in the ITC2007 dataset was reduced from 25.15 to 0.32 by permutation of exam slots and was further improved to 0.14 by reassignments of exams. It is worth noting that for this benchmark problem a second round of slot swapping and exam reassignment resulted in further improvement of cost from 0.14 to 0.13 . However, for most benchmark problems a single round of optimization was sufficient to achieve a competitive exam schedule that could not be improved in the second round.

Table 5. Computational Results of the proposed Approach Applied to the ITC2007 Dataset

\begin{tabular}{|c|c|c|c|c|c|c|c|c|c|c|c|c|c|}
\hline Dataset & $\begin{array}{l}\text { Re- } \\
\text { quired } \\
\text { No of } \\
\text { Slots }\end{array}$ & $\begin{array}{l}\text { Unopti- } \\
\text { mized } \\
\text { Cost }\end{array}$ & $\begin{array}{l}\text { Total } \\
\text { Slots } \\
\text { Conflicts }\end{array}$ & $\begin{array}{l}\text { Minimiza- } \\
\text { tion Of } \\
\text { Slots } \\
\text { Conflicts }\end{array}$ & $\begin{array}{l}\text { Total } \\
\text { Slots } \\
\text { Conflicts }\end{array}$ & $\begin{array}{l}\text { Cost } \\
\text { Before } \\
\text { Further } \\
\text { Optimi- } \\
\text { zation }\end{array}$ & $\begin{array}{l}\text { Re- } \\
\text { assign- } \\
\text { ment } \\
\text { (G:group } \\
\text { /S:single) }\end{array}$ & $\begin{array}{l}\text { Cost } \\
\text { After } \\
\text { Swap } \\
1\end{array}$ & $\begin{array}{l}\text { Cost } \\
\text { After } \\
\text { Reassign } \\
\text { I }\end{array}$ & $\begin{array}{l}\text { Total } \\
\text { Slots } \\
\text { Conflicts }\end{array}$ & $\begin{array}{l}\text { Cost } \\
\text { After } \\
\text { Swap } \\
\text { II }\end{array}$ & $\begin{array}{l}\text { Cost } \\
\text { After } \\
\text { Reassign } \\
\text { II }\end{array}$ & $\begin{array}{l}\text { Total } \\
\text { Slots } \\
\text { Conflicts }\end{array}$ \\
\hline Exam1 & 54 & 23.90 & 7522 & YES & 7414 & 23.49 & $\mathrm{G}$ & 2.02 & 1.12 & 10787 & 1.12 & 1.12 & 10787 \\
\hline Exam2 & 40 & 26.92 & 4740 & YES & 4709 & 26.92 & G & 0.48 & 0.22 & 5359 & 0.22 & 0.22 & 5359 \\
\hline Exam3 & 36 & 28.53 & 9114 & YES & 8928 & 28.53 & G & 3.35 & 1.84 & 12584 & 1.84 & 1.84 & 12584 \\
\hline Exam4 & 21 & 33.84 & 4001 & YES & 3958 & 28.49 & G & 14.62 & 12.06 & 4326 & 12.06 & 12.06 & 4326 \\
\hline Exam5 & 42 & 41.79 & 5156 & YES & 5118 & 41.79 & G & 0.83 & 0.37 & 5736 & 0.37 & 0.37 & 5736 \\
\hline Exam6 & 16 & 13.32 & 1652 & YES & 1647 & 13.32 & G & 5.50 & 4.70 & 1960 & 4.69 & 4.61 & 1954 \\
\hline Exam7 & 80 & 23.38 & 9949 & YES & 9839 & 23.55 & G & 0.16 & 0.07 & 11066 & 0.07 & 0.07 & 11066 \\
\hline Exam8 & 80 & 25.15 & 6843 & YES & 6706 & 25.15 & G & 0.32 & 0.14 & 7374 & 0.13 & 0.13 & 7374 \\
\hline
\end{tabular}

An important feature of the proposed optimization is its deterministic pattern that is preserved for all the datasets. The minimization of the total slots conflicts has proven to be a useful preparatory step for the subsequent minimization of the cost of the exam schedule. By minimizing the slots conflicts we have achieved the greatest "packing" of conflicting exams and, by doing so, have enhanced the possibility of reduction of the schedule cost in subsequent steps. It is worth noting that this is beneficial even if, in some rare circumstances (see Exam7 in the ITC2007 dataset, Table 5; cost increase from 23.38 to 23.55) the reduction of the total slots conflicts comes at the expense of some increase of the schedule cost. This enhanced potential for subsequent reduction of the schedule cost is fully capitalized on in the subsequent step of permutation of exam slots; with the cost reduction to 0.07 .

The permutation of exams slots is a very simple approach and yet it typically produces a very significant reduction of the cost (1) of the initial exam schedule. By splitting the exam scheduling problem into three sub-problems of minimization of slots conflicts, minimization of cost by slot swapping and minimization of cost by reassignments we have achieved a clear deterministic progression of the optimization process that lends itself to easy interpretation.

The reassignments of exams also never fail to reduce the cost (1). Looking into details, group reassignments outperformed single reassignments in most of the datasets. The effect of these reassignments can be seen from the third data point to the fourth data point in each line in the graphs given. There is a very clear pattern, whereby for each line, the graph is going up 
in a diagonal to the left. This means that the exam schedule generated at this stage has a lower cost but from an increase of the overall total slots conflicts.

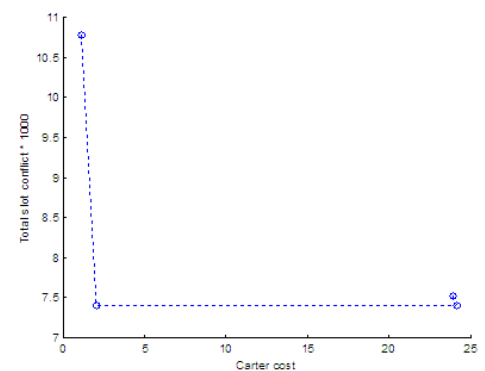

Exam1

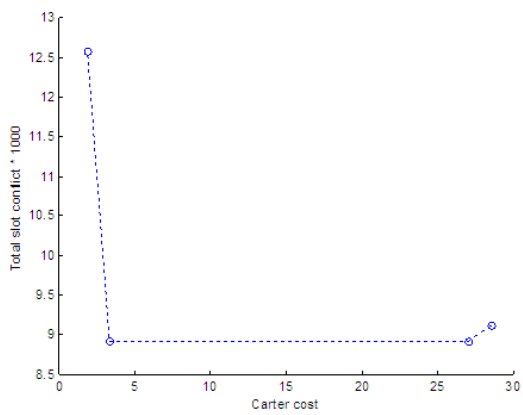

Exam3

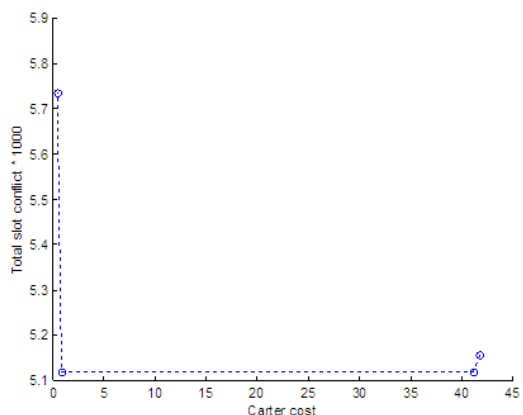

Exam5

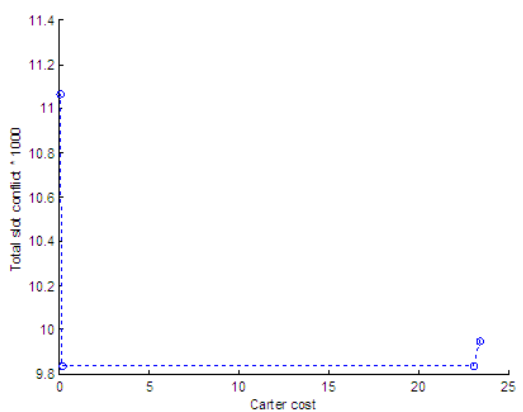

Exam7

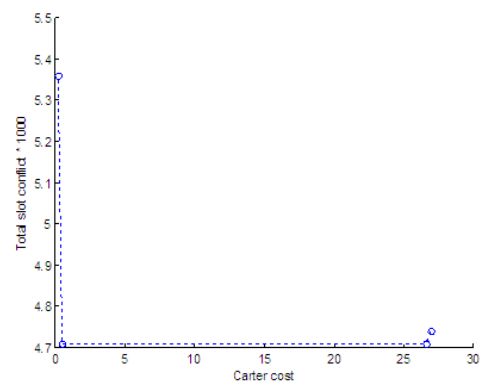

Exam2

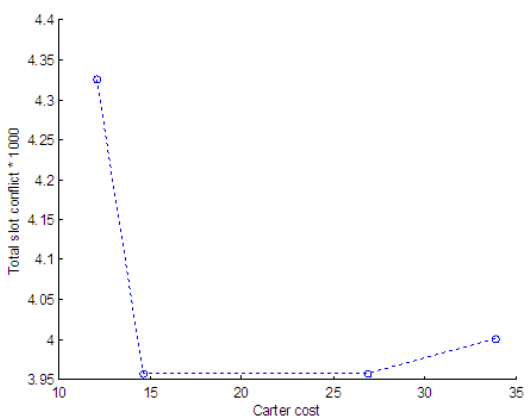

Exam 4

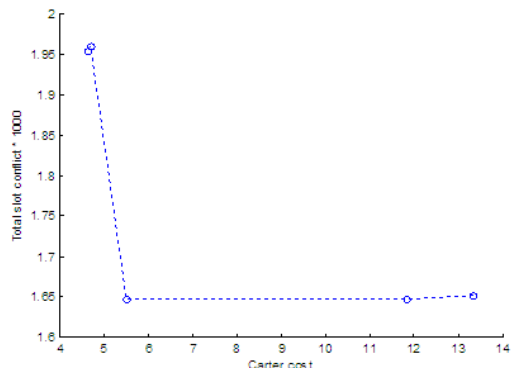

Exam6

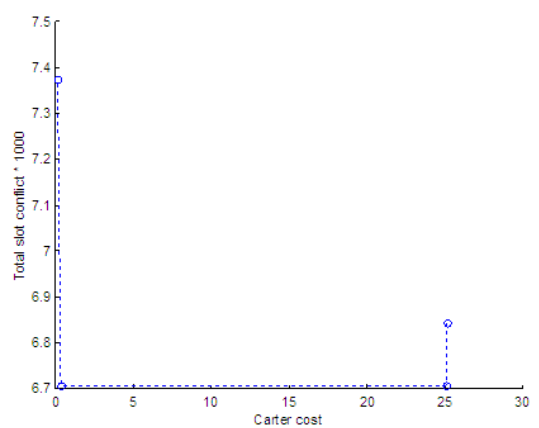

Exam8

Figure 10. Cost (1) vs. the Total Slots Conflicts for ITC2007 Dataset 
While the single reassignment follows the strict minimization of the cost (1), in group reassignment we may benefit from the inherent interaction of effects of reassignment of exams in a group. Although the individual exams in a group have been selected according to their potential to reduce the cost (1), when reassigned to another slot, taken together with other exams in a group, this potential for reduction of cost (1) may be eroded or indeed reversed. Although this is unwelcome, it allows the search to escape from local optima, thus improve on the single reassignment solution. An alternative strategy might be to perform a different type of optimization with single reassignment that would allow the search to escape from local optima (e.g. simulated annealing) but the benefits need to be weighted against computational cost.

It is interesting to note that the feasible solutions with the lower total slots conflicts appear to offer advantage in terms of their increased capacity to minimize the cost (1) through simple re-ordering of slots and subsequently through the re-assignment of exams between slots. However, while at the initial stages of optimization one is justified in making a positive correlation between the cost and the slots conflicts count (as is endorsed by the experiences of other researchers using max-degree pre-ordering of exams in their scheduling heuristics), it is clear that this correlation represents a potential for the reduction of cost by swapping of the slots. At the final stages of our optimization this potential is not relevant as the slots are deemed to have been optimally ordered already. In the rare circumstances where the reassignment of exams creates an opportunity for further cost reduction by swapping the exam slots, a second round of optimization delivers the expected improvement of the exam schedule.

Table 6. Results of Our Method in Comparison with Some Other Constructive Methods in Literature (highlighted columns are for the methods that delivered results for all instances in the Toronto dataset)

\begin{tabular}{|c|c|c|c|c|c|c|c|c|c|}
\hline Dataset & [12] & [9] & [18] & [3] & [21] & [11] & [22] & [23] & Ours \\
\hline car-s-91 (I) & 7.1 & 4.97 & 5.45 & 5.29 & 5.08 & 5.03 & 5.17 & 5.12 & 5.19 \\
\hline car-f-92 (I) & 6.2 & 4.32 & 4.5 & 4.54 & 4.38 & 4.22 & 4.74 & 4.41 & 4.49 \\
\hline ear-f-83(I) & 36.4 & 36.16 & 36.15 & 37.02 & 38.44 & 36.06 & 40.91 & 36.91 & 37.57 \\
\hline hec-s-92(I) & 10.8 & 11.61 & 11.38 & 11.78 & 11.61 & 11.71 & 12.26 & 11.31 & 11.47 \\
\hline kfu-s-93 & 14 & 15.02 & 14.74 & 15.8 & 14.67 & 16.02 & 15.85 & 14.75 & 14.36 \\
\hline lse-f-91 & 10.5 & 10.96 & 10.85 & 12.09 & 11.69 & 11.15 & 12.58 & 11.41 & 11.9 \\
\hline $\begin{array}{l}\text { pur-s-93 } \\
\text { (I) }\end{array}$ & 3.9 & - & - & - & - & - & 5.87 & 5.87 & 4.88 \\
\hline rye-f-92 & 7.3 & - & - & 10.38 & 9.49 & 9.42 & 10.11 & 9.61 & 9.8 \\
\hline sta-f-83(I) & 161.5 & 161.9 & 157.21 & 160.4 & 157.72 & 158.86 & 158.12 & 157.52 & 158.25 \\
\hline tre-s-92 & 9.6 & 8.38 & 8.79 & 8.67 & 8.78 & 8.37 & 9.3 & 8.76 & 8.74 \\
\hline uta-s-92(I) & 3.5 & 3.36 & 3.55 & 3.57 & 3.55 & 3.37 & 3.65 & 3.54 & 3.59 \\
\hline ute-s-92 & 25.8 & 27.41 & 26.68 & 28.07 & 26.63 & 27.99 & 27.71 & 26.25 & 27.37 \\
\hline yor-f-83 (I) & 41.7 & 40.88 & 42.2 & 39.8 & 40.45 & 39.53 & 43.98 & 39.67 & 41.1 \\
\hline
\end{tabular}

A comparison of our results to the results obtained with other constructive methods reported in the literature is presented in Table 6. It is clear that the listed methods have rather uneven performance. They perform well on some benchmark problems and less well on others. This is a rather unwelcome characteristic from the user's perspective, as there is no way of predicting the quality of the solution that will be obtained using a particular method on a new dataset. In this context a mean percentage discrepancy between the solution delivered by a given method and the best solution reported in the literature together with the standard deviation of such discrepancies, evaluated on a representative set of benchmark problems, provide a measure of reliability of the exam scheduling method. In particular, small value of the standard deviation indicates that the method is delivering consistently good results. Table 7 shows that our proposed method is very competitive with the mean percentage discrepancy of $9.11 \%$ between its solutions and the best known ones, and is by far the most consistently reliable, as indicated by the standard deviation 9.77 of these discrepancies. 
Table 7. Average Percentage Distance to the Optimal Cost

\begin{tabular}{|c|c|c|c|c|c|c|c|c|c|}
\hline \multirow[t]{2}{*}{ Dataset } & \multicolumn{2}{|l|}{ [12] } & \multicolumn{2}{|l|}{ [22] } & \multicolumn{2}{|l|}{ [23] } & \multicolumn{2}{|c|}{$\begin{array}{l}\text { Our Proposed } \\
\text { Method }\end{array}$} & \multirow[t]{2}{*}{$\begin{array}{l}\text { Best Constructive } \\
\text { Cost }\end{array}$} \\
\hline & Cost & $\%$ & Cost & $\%$ & Cost & $\%$ & Cost & $\%$ & \\
\hline car-s-91 (I) & 7.10 & 42.86 & 5.17 & 4.02 & 5.12 & 3.02 & 5.19 & 4.43 & 4.97 \\
\hline car-f-92 (I) & 6.20 & 46.92 & 4.74 & 12.32 & 4.41 & 4.50 & 4.49 & 6.40 & 4.22 \\
\hline ear-f-83(I) & 36.40 & 0.94 & 40.91 & 13.45 & 36.91 & 2.36 & 37.57 & 4.19 & 36.06 \\
\hline hec-s-92(I) & 10.80 & 0.00 & 12.26 & 13.52 & 11.31 & 4.72 & 11.47 & 6.20 & 10.80 \\
\hline kfu-s-93 & 14.00 & 0.00 & 15.85 & 13.21 & 14.75 & 5.36 & 14.36 & 2.57 & 14.00 \\
\hline lse-f-91 & 10.50 & 0.00 & 12.58 & 19.81 & 11.41 & 8.67 & 11.90 & 13.33 & 10.50 \\
\hline pur-s-93 (I) & 3.90 & 0.00 & 5.87 & 50.51 & 5.87 & 50.51 & 4.88 & 25.13 & 3.90 \\
\hline rye-f-92 & 7.30 & 0.00 & 10.11 & 38.49 & 9.61 & 31.64 & 9.80 & 34.25 & 7.30 \\
\hline sta-f-83(I) & 161.50 & 2.73 & 158.12 & 0.58 & 157.52 & 0.20 & 158.25 & 0.66 & 157.21 \\
\hline tre-s-92 & 9.60 & 14.70 & 9.30 & 11.11 & 8.76 & 4.66 & 8.74 & 4.42 & 8.37 \\
\hline uta-s-92(I) & 3.50 & 4.17 & 3.65 & 8.63 & 3.54 & 5.36 & 3.59 & 6.85 & 3.36 \\
\hline ute-s-92 & 25.80 & 0.00 & 27.71 & 7.40 & 26.25 & 1.74 & 27.37 & 6.09 & 25.80 \\
\hline yor-f-83 (I) & 41.70 & 5.49 & 43.98 & 11.26 & 39.67 & 0.35 & 41.10 & 3.97 & 39.53 \\
\hline $\begin{array}{l}\text { Average Percentage } \\
\text { Difference To Best } \\
\text { Constructive cost (\%) }\end{array}$ & \multicolumn{2}{|c|}{$\begin{array}{l}\text { mean }=9.06 \\
\text { std }=16.44\end{array}$} & \multicolumn{2}{|c|}{$\begin{array}{l}\text { mean }=15.72 \\
\text { std }=13.84\end{array}$} & \multicolumn{2}{|c|}{$\begin{array}{l}\text { mean }=9.47 \\
\text { std }=14.72\end{array}$} & \multicolumn{2}{|c|}{$\begin{array}{l}\text { mean }=9.11 \\
\text { std }=9.77\end{array}$} & \\
\hline
\end{tabular}

One important point to note when comparing the performance of the various methods is that that several of the best results have been obtained by the methods that did not report any results for pur-f-93 and/or rye-f-92. This is significant because it highlights both the quality, consistency and the universal applicability of our method.

\section{Conclusions and future work}

The proposed optimization approach is very simple yet very competitive in generating reliably high quality exam schedules. We also believe that the domain transformation approach that facilitated transformation of a complex optimization problem into a sequence of more tractable optimizations has a potential for successful applications in a broader spectrum of problems. An important feature of our optimization method is that it preserves the feasibility of the initial solution throughout the whole of the optimization thus saving a considerable computational effort compared to other methods that require customized post-processing.

The proposed method is also very reliable and stable in producing schedules on larger problem instances, for instance pur-s-93 in the Toronto dataset and Exam7 in the ITC2007 dataset.

We expect that the proposed method can be adapted in a relatively straightforward manner to the capacitated scheduling problem by introducing appropriate granular data structures that will permit the requited domain transformation in the optimization process. Also, other constraints suggested at the 2nd International Timetabling Competition in 2007-08 should fit into the general framework of the proposed method.

\section{References}

[1] Argile A., Peytchev E., Bargiela A., Kosonen I., DIME: A Shared Memory Environment for Distributed Simulation, Monitoring and Control of Urban Traffic, Proceedings of European Simulation Symposium ESS'96, Geno. 1996; 152-156.

[2] Asmuni H., Burke, E. K., Garibaldi J. M., and McCollum B. Fuzzy Multiple Heuristic Orderings for Examination Timetabling. In E. K. Burke and M. Trick, editors, volume 3616 of Lecture Notes in Computer Science. Berlin, Springer. 2005; $334-353$. 
[3] Asmuni H, Burke E. K., Garibaldi J. M., McCollum B. and Parkes A. J. An investigation of fuzzy multiple heuristic orderings in the construction of university examination timetables. Comput. Oper. Res. 2009; 36: 981-1001. http://dx.doi.org/10.1016/j.cor.2007.12.007

[4] Bargiela A., An algorithm for observability determination in water-system state estimation, IEE Proceedings Part D, 132. 1985; 245-249.

[5] Bargiela A., Pedrycz W., Hirota K., Granular prototyping in fuzzy clustering, IEEE Transactions on Fuzzy Systems. 2004; 12(5): 697-709. http://dx.doi.org/10.1109/TFUZZ.2004.834808

[6] Bargiela, A., and Pedrycz, W. Granular Computing - An Introduction, Kluwer Academic Publishers. 2002. http://dx.doi.org/10.1007/978-1-4615-1033-8

[7] Bargiela, A. and Pedrycz, W. Toward a theory of Granular Computing for human-centred information processing, IEEE Trans. on Fuzzy Systems. 2008; 16(2): 320-330. http://dx.doi.org/10.1109/TFUZZ.2007.905912

[8] Burke E.K., Bykov Y., Newall J. and Petrovic S. A Time-Predefined Local Search Approach to Exam Timetabling Problems. IIE Transactions on Operations Engineering. 2004; 36(6): 509-528.

[9] Burke E.K. and J. P. Newall. Solving examination timetabling problems through adaptation of heuristics orderings. Ann. Oper. Res. 2004; 129: 107-134. http://dx.doi.org/10.1023/B:ANOR.0000030684.30824.08

[10] Burke E.K., A. Meisels, S. Petrovic and R. Qu. A graph-based hyperheuristic for educational timetabling problem. European J. Oper. Res. 2007; 176: 177-192. 2007. http://dx.doi.org/10.1016/j.ejor.2005.08.012

[11] Burke E.K., Pham N, Qu R, Yellen J. Linear Combinations of Heuristics for Examination Timetabling. Annals of Operations Research. 2010. http://dx.doi.org/10.1007/s10479-011-0854-y

[12] Carter M., Laporte G. and Lee S. Examination Timetabling: Algorithmic Strategies and Applications. Journal of Operations Research Society. 1996; 47: 373-383.

[13] Carter M. and Laporte G. Recent developments in practical examination timetabling. Lecture Notes in Comput. Sci. 1995; 1153:1-21.

[14] Dowsland K.A. and Thompson J. Ant colony optimization for the examination scheduling problem. Journal of Operational Research Society. 2005; 56: 426-438. http://dx.doi.org/10.1057/palgrave.jors.2601830

[15] Gueret C., Jussien N., Boizumault P., Prins C. Building University Timetables Using Constraint Logic Programming. First International Conference on the Practice and Theory of Automated Timetabling, PATAT. 1995; 95: 393-408, Edinburgh. 1995.

[16] Pedrycz W, Smith M.H., Bargiela A, Granular signature of data, Proc. 19th Int. (IEEE) Conf. NAFIPS'2000, Atlanta. July 2000; 69-73. http://dx.doi.org/10.1109/NAFIPS.2000.877387

[17] Peytchev E., Bargiela A., Gessing R., A Predictive Macroscopic City Traffic Flows Simulation Model, Proceedings of European Simulation Symposium ESS'96, Genoa, ISBN 1-565555-099-4. October 1996; 2: 38-42.

[18] Qu R, Burke EK. Adaptive decomposition and construction for examination timetabling problems. In: In Multidisciplinary international scheduling: theory and applications (MISTA'07), Paris, France. 2007; 418-425.

[19] Qu. R, Burke E.K., B. McCollum, L.T.G. Merlot, and S. Y. Lee. A Survey of Search Methodologies and Automated System Development for Examination Timetabling. Journal of Scheduling. 2009; 12(1): 55-89. http://dx.doi.org/10.1007/s10951-008-0077-5

[20] Rahim, S. K. N. A., Bargiela, A., \& Qu, R. Granular Modelling Of Exam To Slot Allocation. ECMS 2009 Proceedings edited by J. Otamendi, A. Bargiela, J. L. Montes, L. M. Doncel Pedrera. European Council for Modeling and Simulation. 2009; 861-866. http://dx.doi.org/10.7148/2009-0861-0866

[21] Rahman S.A., Bargiela A, E. K. Burke, B. McCollum, and E. Ozcan. Construction of examination timetables based on ordering heuristics. In Proceedings of the 24th International Symposium on Computer and Information Sciences. 2009; 727-732. http://dx.doi.org/10.1109/ISCIS.2009.5291905

[22] Rahman S.A, S., Burke E.K., Bargiela A., McCollum B., Ozcan E. A Constructive approach to examination timetabling based on adaptive decomposition and ordering, Annals of Operation Research. 2011a; 1-19. http://dx.doi.org/10.1007/s10479-011-0999-8

[23] Rahman S.A, Bargiela A., Burke E.K., Ozcan E., McCollum B. Linear combination of heuristic orderings in construction of examination timetable, European Journal of Operational Research.2013. In press.

[24] Rao S. S. Engineering Optimization - Theory and Practice (3rd Edition) [Internet]. (pp: 5). John Wiley \& Sons. Available from: http://knovel.com/web/portal/browse/display?_EXT_KNOVEL_DISPLAY_bookid=1100\&VerticalID=0

[25] Gani T. A., Khader A.T. and Budiarto R. Optimizing Examination Timetabling using a Hybrid Evolution Strategies. In: Proceedings of the Second International Conference on Autonomous Robots and Agents (ICARA 2004), 13-15 December 2004, Palmerston North, New Zealand. 2004; 345-349. 